\title{
Inclusion-Based Approximate Reasoning
}

\author{
Chris Cornelis ${ }^{1}$ and Etienne E. Kerre ${ }^{1}$ \\ Department of Mathematics and Computer Science, Ghent University \\ Fuzziness and Uncertainty Modelling Research Unit \\ Krijgslaan 281 (S9), B-9000 Gent, Belgium \\ \{chris.cornelis, etienne.kerre\} Orug.ac.be \\ WWW homepage: http://allserv.rug.ac.be/ ekerre
}

\begin{abstract}
We present a kind of fuzzy reasoning, dependent on a measure of fulfilment of the antecedent clause, that captures all the expressiveness of the traditional approximate reasoning methodology based on the Compositional Rule of Inference (CRI) and at the same time rules out a good deal of its inherent complexity. We also argue why this approach offers a more genuine solution to the implementation of analogical reasoning than the classically proposed similarity measures.
\end{abstract}

\section{Introduction and Preliminaries}

Reasoning with imprecise information expressed as fuzzy sets has received an enormous amount of attention over the last 30 years. More specifically, researchers have undertaken various attempts to model the following reasoning scheme (an extension of the modus ponens logical deduction rule), known as Generalized Modus Ponens (GMP):

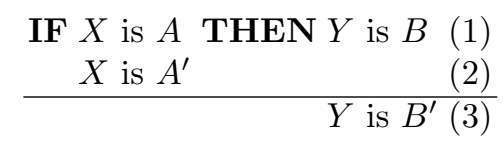

where $X$ and $Y$ are assumed to be variables taking values in the respective universes $U$ and $V$; furthermore $A, A^{\prime} \in \mathcal{F}(U)$ and $B, B^{\prime} \in \mathcal{F}(V) \sqrt{1]}$.

Zadeh suggested to model the if-then rule (1) as a fuzzy relation $R$ (a fuzzy set on $U \times V$ ) and to apply the Compositional Rule of Inference (CRI) to yield an inference about $Y$. The CRI is the following inference pattern:

$$
\begin{aligned}
& X \text { and } Y \text { are } R \\
& X \text { is } A^{\prime} \\
& Y \text { is } R \circ_{T} A^{\prime}
\end{aligned}
$$

where ${ }^{\circ} T$ represents the fuzzy composition of $R$ and $A^{\prime}$ by the $t-$ norm $2^{2} T$, i. e. for every $v \in V$ we have:

\footnotetext{
${ }^{1}$ By $\mathcal{F}(U)$ we denote all fuzzy sets in a universe $U$.

${ }^{2}$ A $t$-norm is any symmetric, associative, increasing $[0,1] \times[0,1] \rightarrow[0,1]$ mapping $T$ satisfying $T(1, x)=x$ for every $x \in[0,1]$ 


$$
B^{\prime}(v)=\sup _{u \in U} T\left(A^{\prime}(u), R(u, v)\right) .
$$

We will refer to the above approach as CRI-GMP, i.e. an implementation of GMP by CRI.

It is obvious that in order for CRI-GMP inference to be reasonable, $R$ and $T$ can not be just any combination of a fuzzy relation and a $t$-norm. One important way of determining the soundness of an inference strategy is to check whether it complies with certain desirable properties of inference in classical logic. For example, we might impose the following (non-exhaustive) list of criteria:

$$
\begin{array}{ll}
\text { A.1 } B \subseteq B^{\prime} & \text { (nothing better than } B \text { can be inferred) } \\
\text { A.2 } A_{1}^{\prime} \subseteq A_{2}^{\prime} \Rightarrow B_{1}^{\prime} \subseteq B_{2}^{\prime} & \text { (monotonicity) } \\
\text { A.3 } A^{\prime}=A \Rightarrow B^{\prime}=B & \text { (compatibility with modus ponens) } \\
\text { A.4 } A^{\prime} \subseteq A \Rightarrow B^{\prime}=B & \text { (fulfilment of } A \text { implies fulfilment of } B \text { ) }
\end{array}
$$

The first three are all standard in the approximate reasoning literature (see e.g. [1] 6]); we have added the fourth ${ }^{3}$ as a straightforward extension of a commonsense principle that stems from the following intuition: the antecedent of a rule represents a restriction enforced on a variable, and the fulfilment of this restriction (this happens whenever $A^{\prime} \subseteq A$ ) is a sufficient condition for the fulfilment of the consequent. This is exemplified by the crisp rule "Any Belgian older than 18 is entitled to get his driving licence". To conclude that a person may apply for a licence, it suffices to know that his age is restricted to belong to a subset of $[18,+\infty[$.

Noticing the link with classical logic, authors have proposed various fuzzifications of the implication operator to model $R$. A very general definition of a so-called implicator is the following:

Definition 1.1. (Implicator) Any $[0,1]^{2} \rightarrow[0,1]$-operator $\mathcal{I}$ of which the first and second partial mappings are decreasing, respectively increasing, and so that $\mathcal{I}(0,0)=\mathcal{I}(0,1)=\mathcal{I}(1,1)=1$ and $\mathcal{I}(1,0)=0$ is called an implicator.

Table 1 lists some common implicators. After choosing an implicator $\mathcal{I}$, we put $R(u, v)=\mathcal{I}(A(u), B(v))$ for all $(u, v) \in U \times V$.

The suitability of a given $(T, \mathcal{I})$ pair to implement the CRI-GMP can then be evaluated with respect to the listed criteria; extensive studies exist on this issue (see e.g. [6]).

One particularly unfortunate aspect of the CRI is its high complexity. In general, for finite universes $U$ and $V$ so that $|U|=m$ and $|V|=n$, an inference requires $O(m n)$ operations. Mainly for this reason, researchers have explored

\footnotetext{
${ }^{3}$ Strictly speaking, A.4 is superfluous, as it is a direct consequence of A.1, A.2 and
} A.3 combined. We explicitly mention it here because of its intuitive logical appeal. 
Table 1. Implicators on the unit interval $\left((x, y) \in[0,1]^{2}\right)$

\begin{tabular}{|c|l|l|}
\hline Symbol & Name & Definition \\
\hline \hline $\mathcal{I}_{m}$ & Zadeh & $\mathcal{I}_{m}(x, y)=\max (1-x, \min (x, y))$ \\
$\mathcal{I}_{a}$ & Eukasiewicz & $\mathcal{I}_{a}(x, y)=\min (1,1-x+y)$ \\
$\mathcal{I}_{g}$ & Gödel & $\mathcal{I}_{g}(x, y)=\left\{\begin{array}{l}1 \text { if } x \leq y \\
y \text { otherwise }\end{array}\right.$ \\
$\mathcal{I}_{b}$ & Kleene-Dienes & $\mathcal{I}_{b}(x, y)=\max (1-x, y)$ \\
$\mathcal{I}_{\Delta}$ & Goguen & $\mathcal{I}_{\Delta}(x, y)=\left\{\begin{array}{l}1 \text { if } x \leq y \\
\frac{y}{x} \text { otherwise }\end{array}\right.$ \\
\hline
\end{tabular}

other ways of performing fuzzy inference while preserving its useful characteristics. In the next sections, we will propose an alternative based on a fuzzification of crisp inclusion, and argue why it is better than the existing methods.

\section{Inclusion-Based Approach}

From the criteria listed in the introduction, it is clear that when the observation $A^{\prime}$ of $X$ is a subset of $A$, the restriction on $Y$ should be exactly $B$ by A.4. On the other hand, as $A^{\prime}$ moves away from ("is less included in", "less fulfills") $A$, by A.1 the imposed restriction on $Y$ can only get weaker, meaning that we will be able to infer less and less information. Bearing in mind the close relationship between fulfilment and inclusion, we might capture this behaviour provided we can somehow measure the degree of inclusion of $A^{\prime}$ into $A$.

Indeed, if we have such a measure (say $\operatorname{Inc}\left(A^{\prime}, A\right)$ ) at our disposal, we can use it to transform the consequent fuzzy set $B$ into an appropriate $B^{\prime}$. Schematically, this amounts to the following:

$$
\begin{aligned}
& \text { IF } X \text { is } A \text { THEN } Y \text { is } B \\
& X \text { is } A^{\prime} \\
& \hline Y \text { is } f\left(\operatorname{Inc}\left(A, A^{\prime}\right), B\right)
\end{aligned}
$$

Good candidates for the $(f, I n c)$ pair will preferably be such that A.1 through A.4 hold with as little extra conditions added as possible. In addition, we would like to have $\operatorname{Inc}\left(B^{\prime}, B\right)=\operatorname{Inc}\left(A^{\prime}, A\right)$, in order that a kind of symmetry between the fulfilment of $B^{\prime}$ by $B$ and that of $A^{\prime}$ by $A$ is respected. In this section and the next, we will consider each of these problems in turn.

\subsection{Fuzzification of Set Inclusion}

As has been done for just about every aspect of classical set theory and binary logic, several authors have introduced frameworks for extending the subsethood notion to fuzzy sets. For a long time Zadeh's definition of inclusion, which reads 


$$
A \subseteq B \Longleftrightarrow(\forall u \in U)(A(u) \leq B(u)),
$$

remained unquestioned by the fuzzy community. Bandler and Kohout were among the first to contest this naive view, terming it "an unconscious step backward in the realm of dichotomy". They commented on the relationship between implication and inclusion, arguing that in classical set theory $A$ is a subset of $B$ $(A, B$ defined in a universe $U)$ if and only if

$$
(\forall u \in U)(u \in A \Rightarrow u \in B)
$$

For each element $u$, we check whether $u \in A$. If so, then $u$ must also be in $B$. Otherwise, we enforce no constraint on the membership of $u$ to $B$. Bandler and Kohout suggested the following fuzzy extension of this mathematical principle (I being an implicator):

$$
\operatorname{Inc}_{\mathcal{I}}(A, B)=\inf _{u \in U} \mathcal{I}(A(u), B(u)) .
$$

As there are various implicators with varying suitability, we also have the choice between a multitude of inclusion operators which have or have not certain desired properties. We have adapted a list of selected properties taken from [10]:

$$
\begin{aligned}
& \text { I.1 } A \subseteq B \Longleftrightarrow \operatorname{Inc}_{\mathcal{I}}(A, B)=1 \\
& \text { I.2 }(\exists u \in U)(A(u)=1 \text { and } B(v)=0) \Longleftrightarrow \operatorname{Inc}_{\mathcal{I}}(A, B)=0 \\
& \text { I.3 } B \subseteq C \Rightarrow I n c_{\mathcal{I}}(C, A) \leq I n c_{\mathcal{I}}(B, A) \\
& \text { I.4 } B \subseteq C \Rightarrow I n c_{\mathcal{I}}(A, B) \leq \operatorname{Inc_{\mathcal {I}}}(A, C) \\
& \text { I.5 } \operatorname{Inc_{\mathcal {I}}}(A, B)=\operatorname{Inc} c_{\mathcal{I}}(\operatorname{co} B, \operatorname{co} A) \\
& \text { I.6 } I n c_{\mathcal{I}}(B \cup C, A)=\min \left(I n c_{\mathcal{I}}(B, A), I n c_{\mathcal{I}}(C, A)\right) \\
& \text { I.7 } I n c_{\mathcal{I}}(A, B \cap C)=\min \left(I n c_{\mathcal{I}}(A, B), \operatorname{Inc_{\mathcal {I}}}(A, C)\right) \\
& \text { I. } 8 \operatorname{Inc}_{\mathcal{I}}(A, C) \geq \min \left(I n c_{\mathcal{I}}(A, B), \operatorname{Inc} c_{\mathcal{I}}(B, C)\right)
\end{aligned}
$$

Criterion I.2 might at first glance seem harsh (e.g. Wilmott [12] and Young [13] preferred to leave it out in favour of more compensating operators), but as Sinha and Dougherty [8] proved, it is indispensible if we want $I n c_{\mathcal{I}}$ to be a faithful extension of the classical inclusion, that is, $\operatorname{Inc}_{\mathcal{I}}(A, B) \in\{0,1\}$ if $A$ and $B$ are crisp sets.

Regarding these conditions we may prove the following theorem: [10]

\section{Theorem 2.1. (Properties of $I n c_{\mathcal{I}}$ )}

1. Inc $c_{\mathcal{I}}$ always satisfies I.3, I.4, I.6, I.7 and the sufficient part of I.2

2. If $\mathcal{I}$ satisfies $\left(\forall(x, y) \in[0,1]^{2}\right)(x \leq y \Longleftrightarrow \mathcal{I}(x, y)=1)$, then Inc $\mathcal{I}_{\mathcal{I}}$ satisfies I. 1

3. If $\mathcal{I}$ satisfies $\left(\forall(x, y) \in[0,1]^{2}\right)(\mathcal{I}(x, y)=\mathcal{I}(1-y, 1-x))$, then Inc I $_{\mathcal{I}}$ satisfies I.5

4. If $\mathcal{I}$ is transitive, then Inc $_{\mathcal{I}}$ satisfies $I .8$ 
It turns out that we can test $I n c_{\mathcal{I}}$ for suitability by checking just the discriminating conditions I.1, (the necessary part of) I.2, I.5 and I.8. For instance, $I n c_{\mathcal{I}_{a}}$ satisfies all conditions (including the necessary part of I.2 provided the universe is finite) [10] 11], while $I n c_{\mathcal{I}_{b}}$ does not since the Kleene-Dienes implicator is neither contrapositive nor transitive.

\subsection{Modification of the Consequent}

After evaluating $\alpha=\operatorname{Inc}\left(A^{\prime}, A\right)$ by a chosen inclusion measure, we proceed by modifying $B$ into a suitable output $B^{\prime}$, i.e. $B^{\prime}(v)=f(\alpha, B)(v)$ for all $v$ in $V$. To comply with condition A.1, it is clear that $f(\alpha, B)(v) \geq B(v)$. On the other hand, assuming Inc satisfies the monotonicity condition I.4, $f$ ought to be increasing w.r.t. its first argument to fulfil A.2. Lastly, to have A.3 and A.4 it is mandatory that $f(1, B)=B$, whatever $B \in \mathcal{F}(V)$.

The need for modification mappings also arises in similarity-based reasoning, where instead $\alpha$ will result from a similarity measurement, so we can "borrow", so to speak, some of the work that has been done in that field. For instance, Türkşen and Zhong [9] use the following forms, for all $v \in V$ :

$$
\begin{aligned}
& f_{1}(\alpha, B)(v)= \begin{cases}\min \left(1, \frac{B(v)}{\alpha}\right) & \text { if } \alpha>0 \\
1 & \text { otherwise }\end{cases} \\
& f_{2}(\alpha, B)(v)=\alpha B(v)
\end{aligned}
$$

$f_{2}$ drops out immediately because it violates A.1. $f_{1}$ on the other hand does obey our postulates, and so does the following mapping:

$$
f_{3}(\alpha, B)(v)=B(v)^{\alpha}
$$

for all $v \in V$. Another alternative, adopted from Bouchon-Meunier and Valverde [4], introduces a level of uncertainty proportional to $1-\alpha$, thus making inference results easy to interpret: the higher this value gets, the more the original $B$ is "flooded" by letting the minimal membership grade in $B^{\prime}$ for every $v$ in $V$ become at least $1-\alpha$ :

$$
f_{4}(\alpha, B)(v)=\max (1-\alpha, B(v))
$$

We thus observe that several modification mappings serve our cause; deciding which one to choose depends largely on the application at hand. Nevertheless, in a situation where we would like the inference result to be in accordance somehow with the output of a given CRI-GMP system, one mapping might be considered more eligible than the next one. This will be clarified in the next section.

\section{Link with the Compositional Rule of Inference}

In the previous sections we showed how inclusion could be related to implication. It is not surprising, then, to discover that the behaviour of the CRI-GMP based 
on particular $t$-norm/implicator pairs can be linked to our inclusion approach. In fact, it can serve as a benchmark for testing the quality as well as the soundness of our proposed inference system.

In particular, we may show that for a residuated 4 implicator generated by a continuous $t$-norm $T$, the following theorem holds:

Theorem 3.1. Let $T$ be a continuous $t-n o r m$. If $B^{\prime}$ represents the result obtained with $C R I-G M P$ based on the $\left(T, \mathcal{I}_{T}\right)$ pair, i.e. for all $v \in V$

$$
B^{\prime}(v)=\sup _{u \in U} T\left(A^{\prime}(u), \mathcal{I}_{T}(A(u), B(v))\right),
$$

then

$$
\operatorname{Inc}_{\mathcal{I}_{T}}\left(B^{\prime}, B\right) \geq \operatorname{Inc}_{\mathcal{I}_{T}}\left(A^{\prime}, A\right)
$$

Additionally, if $(\forall \alpha \in[0,1])(\exists v \in V)(B(v)=\alpha)$, then

$$
\operatorname{Inc}_{\mathcal{I}_{T}}\left(B^{\prime}, B\right)=\operatorname{Inc}_{\mathcal{I}_{T}}\left(A^{\prime}, A\right)
$$

Proof. This proof is based on properties of residuated implicators generated by continuous $t$-norms as listed by Klir and Yuan: 7]

$$
\begin{aligned}
& \text { I } \quad \mathcal{I}_{T}\left(\sup _{j \in J} a_{j}, b\right)=\inf _{j \in J} \mathcal{I}_{T}\left(a_{j}, b\right) \\
& \text { II } \mathcal{I}_{T}(T(a, b), d)=\mathcal{I}_{T}\left(a, \mathcal{I}_{T}(b, d)\right) \\
& \text { III } \mathcal{I}_{T}\left(\mathcal{I}_{T}(a, b), b\right) \geq a \\
& \text { IV } T(a, b) \leq d \Longleftrightarrow \mathcal{I}_{T}(a, d) \geq b
\end{aligned}
$$

where $J$ is an arbitrary index set, $\left(a_{j}\right)_{j \in J}$ a family in $[0,1], a, b, d \in[0,1]$.

$$
\begin{aligned}
& I n c_{\mathcal{I}_{T}}\left(B^{\prime}, B\right)=\inf _{v \in V} \mathcal{I}_{T}\left(B^{\prime}(v), B(v)\right) \\
& =\inf _{v \in V} \mathcal{I}_{T}\left(\sup _{u \in U} T\left(A^{\prime}(u), \mathcal{I}_{T}(A(u), B(v))\right), B(v)\right) \\
& =\inf _{v \in V} \inf _{u \in U} \mathcal{I}_{T}\left(T\left(A^{\prime}(u), \mathcal{I}_{T}(A(u), B(v))\right), B(v)\right) \text { (Property I) } \\
& =\inf _{v \in V} \inf _{u \in U} \mathcal{I}_{T}\left(A^{\prime}(u), \mathcal{I}_{T}\left(\mathcal{I}_{T}(A(u), B(v)), B(v)\right)\right) \text { (Property II) } \\
& \geq \inf _{v \in V} \inf _{u \in U} \mathcal{I}_{T}\left(A^{\prime}(u), A(u)\right) \\
& =\operatorname{Inc}_{\mathcal{I}_{T}}\left(A^{\prime}, A\right)
\end{aligned}
$$

In the one but last step of this proof, if we can guarantee that there exists a $v \in V$ so that $B(v)=A(u)$, then for this value the infimum over all $v \in V$ is reached (considering that $\mathcal{I}_{T}\left(\mathcal{I}_{T}(A(u), A(u)), A(u)\right)=\mathcal{I}_{T}(1, A(u))=A(u)$ and that the second partial mapping of $\mathcal{I}_{T}$ is increasing).

\footnotetext{
${ }^{4}$ Any $t$-norm $T$ generates a so-called residuated implicator $\mathcal{I}_{T}$ by the formula $\mathcal{I}_{T}(x, y)=\sup \{\gamma \mid \gamma \in[0,1]$ and $T(x, \gamma) \leq y\}[10$
} 
Corollary 3.1. For every $v \in V$, the inference result $B^{\prime}(v)$ obtained with $C R I-$ $G M P$ based on the $\left(T, \mathcal{I}_{T}\right)$ pair, where $T$ is a continuous $t-n o r m$, is bounded above by the expression $\mathcal{I}_{T}\left(\operatorname{Inc}_{\mathcal{I}_{T}}\left(A^{\prime}, A\right), B(v)\right)$.

Proof. Suppose $\operatorname{Inc}_{\mathcal{I}_{T}}\left(A^{\prime}, A\right)=\alpha$. From theorem 3.1 we conclude that $B^{\prime} \in\left\{B^{*} \in \mathcal{F}(V) \mid \inf _{v \in V} \mathcal{I}_{T}\left(B^{*}(v), B(v)\right) \geq \alpha\right\}$. Hence, for all $v \in V$, we obtain successively:

$$
\begin{aligned}
\mathcal{I}_{T}\left(B^{\prime}(v), B(v)\right) \geq \alpha & \Longleftrightarrow T\left(B^{\prime}(v), \alpha\right) \leq B(v) \quad \text { (Property IV) } \\
& \left.\Longleftrightarrow T\left(\alpha, B^{\prime}(v)\right) \leq B(v) \quad \text { (Symmetry of } T\right) \\
& \Longleftrightarrow \mathcal{I}_{T}(\alpha, B(v)) \geq B^{\prime}(v) \text { (Property IV) }
\end{aligned}
$$

This completes the proof.

In effect, this shows that if we put $f(\alpha, B)(v)=\mathcal{I}_{T}(\alpha, B(v))$ for every $v$ in $V$, a conclusion entailed by our algorithm is a superset (not necessarily a proper one) of the according CRI-GMP result, which can be regarded as a justification of its soundness: when we replace the output of the CRI-GMP by a less specific fuzzy set, the result will never contain more information than we were allowed to deduce with the original (reliable) inference mechanism.

We will make these results concrete by applying them to the Lukasiewicz implicator 15 , of which we already know (from section 2) that the associated inclusion measure excels w.r.t. the proposed axioms. In this case, $\alpha=I n c_{\mathcal{I}_{a}}\left(A^{\prime}, A\right)=$ $\inf _{u \in U} \min \left(1,1-A^{\prime}(u)+A(u)\right)$ and according to corollary $3.1 f(\alpha, B)(v)=$ $\min (1,1-\alpha+B(v))$ imposes itself as the proper choice of modification mapping.

Example 3.1. Consider the rule

IF dirt level is low THEN washing time is medium

which could be part of the implementation of a simple fuzzy washing machine. We agree to express $X$ (dirt level) in percentages, while the universe of $Y$ (washing time) is taken to be the real interval [0,90]. For simplicity, all membership functions have triangular shape.

We illustrate the results of our approach to several fuzzy inputs. Figure 1 shows the membership functions for "low" and "medium". The first input in figure 2 could be interpreted to mean "somewhat low", while the second may represent the concept "around 20". It can be checked that $I n c_{\mathcal{I}_{a}}$ ( somewhat low,low) $=$ 0.66 and $I n c_{\mathcal{I}_{a}}$ (around 20,low) $=0.60$.

In figure 3 we show the inference result obtained with the inclusion-based approach. Each picture also shows, as a dashed line, the output generated by the corresponding CRI-GMP method (i.e. the one based on the $\left(W, \mathcal{I}_{a}\right)$ pair).

\footnotetext{
${ }^{5} \mathcal{I}_{a}$ is in fact a residual implicator generated by the $t$-norm $W$ defined as $W(x, y)=$ $\max (0, x+y-1),(x, y) \in[0,1]^{2}$
} 
Notice that, while the maximal difference between the CRI-GMP result and ours is bounded above by $1-I n c_{\mathcal{I}_{a}}\left(A^{\prime}, A\right)\left(\right.$ since $B(v) \leq B^{\prime}(v) \leq f(\alpha, B)(v)$ ), we generally get a fairly tight upper approximation and in a lot of cases, as 3.b exemplifies, the results are equal.

As far as efficiency is concerned, the inclusion method clearly outperforms the CRI-GMP: our technique requires only one inf-calculation (one min-calculation in a finite universe) per inference while in the latter case we need to perform this tedious operation for every $v \in V$.
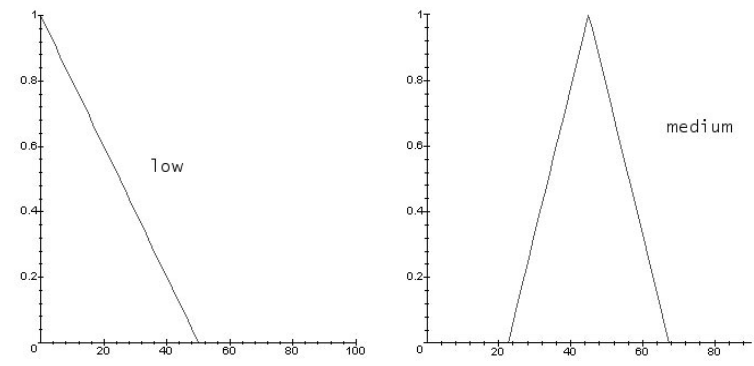

Fig. 1. a) concept "low" b) concept "medium"
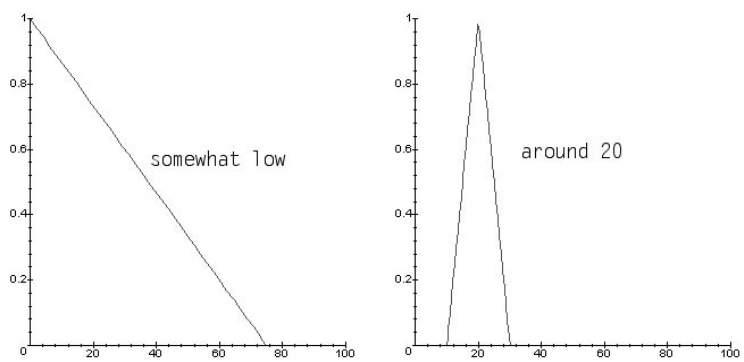

Fig. 2. a) concept "somewhat low" b) concept "around 20"

\section{Relationship to Analogical Reasoning}

Several authors have pursued the strategy of analogical reasoning in their quest to model human behaviour in various cognitive tasks, such as classification and decision making. As Bouchon et al. [3] state, "it amounts to inferring, from a 

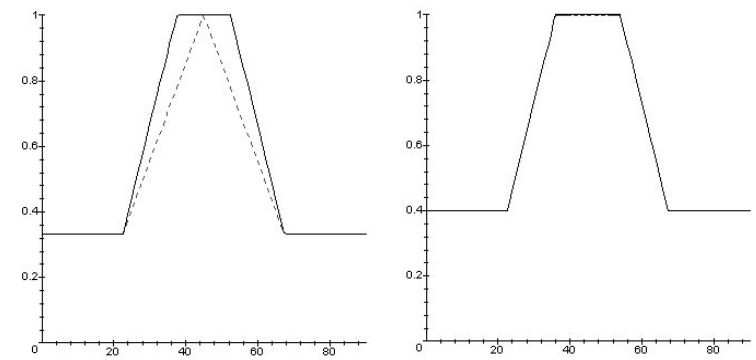

Fig. 3. a) inference for "somewhat low" b) inference for "around 20"

known case which is similar enough to the encountered situation, that what is true in the known case might still be true or approximately true in this situation".

A panoply of similarity measures have been reviewed in the literature as a means to draw analogies between situations (fuzzy sets). Just like our inclusion measures, these operators yield a [0,1]-valued degree for any two fuzzy sets in a given universe, which can likewise be used to set up appropriate modification mappings. Similarity is however not a uniquely defined notion, and care should be taken when adopting such or such interpretation for use in an application. The predominant characteristics that most people look for in a similarity measure $\mathcal{S}$ seem to be those proposed by Zadeh [14, i.e6

$$
\begin{array}{ll}
\mathrm{S} .1 \mathcal{S}(A, A)=1 & \text { (reflexivity) } \\
\mathrm{S} .2 \mathcal{S}(A, B)=\mathcal{S}(B, A) & \text { (symmetry) } \\
\mathrm{S} .3 \min (\mathcal{S}(A, B), \mathcal{S}(B, C)) \leq \mathcal{S}(A, C) & \text { (min-transitivity) }
\end{array}
$$

where $A, B$ and $C$ are fuzzy sets in a given universe $U$.

For our purposes, i.e. the modelling of approximate analogical reasoning, symmetry is actually both counterintuitive and harmful! Counterintuitive, because we compare an observation $A^{\prime}$ to a reference $A$ and not the other way round; harmful, because imposing symmetry inevitably clashes with the soundness condition A.4 (from $A^{\prime} \subseteq A$ infer $B^{\prime}=B$ ), and renders inference absolutely useless (just imagine a symmetrical measure $\mathcal{S}$ that satisfies $\mathcal{S}(A, B)=1$ if $A \subseteq B)$. Still, most of the research done so far did not take heed of this evident pitfall, with the notable exception of Bouchon-Meunier and Valverde [4]: they are also inclined to drop the symmetry requirement, but they failed to link their proposed operators to the intuitive notion of fulfilment that we have developed in the course of this paper.

So while we see that the idea of analogical reasoning is in itself very useful, its execution so far has often been subject to essential misconceptions that have put it, from a logical perspective, on the wrong track.

\footnotetext{
${ }^{6}$ The transitivity condition S.3 can be relaxed by allowing a general $t$-norm to replace $\min$
} 


\section{Conclusion}

Our aim in this paper was twofold: firstly, we set out to reduce the complexity present in the CRI-GMP, and secondly we redefined the semantics of analogical reasoning in terms of fulfilment, stressing that the traditional symmetry condition is not only superfluous but also harmful to the proper functioning of an inference system.

\section{Acknowledgements}

Chris Cornelis would like to acknowledge the Fund for Scientific Research Flanders (FWO) for supporting the research elaborated on in this paper.

\section{References}

1. Baldwin J.F., Pilsworth, B.: Axiomatic approach to implication for approximate reasoning using fuzzy logic. Fuzzy Sets and Systems 3 (1980) 193-219

2. Bandler W., Kohout, L.: Fuzzy power sets and fuzzy implication operators. Fuzzy Sets and Systems 4 (1980), 13-30

3. Bouchon-Meunier B., Dubois D., Godo L., Prade, H.: Fuzzy sets and possibility theory in approximate and plausible reasoning. Fuzzy sets in approximate reasoning and information systems, Kluwer Academic Publishers. (1999) 15-190

4. Bouchon-Meunier B., Valverde L.: A fuzzy approach to analogical reasoning. Soft Computing 3 (1999) 141-147

5. Cornelis C., De Cock M., Kerre E.E.: The generalized modus ponens in a fuzzy set theoretical framework. Fuzzy IF-THEN Rules in Computational Intelligence, Theory and Applications (eds. D. Ruan en E. E. Kerre), Kluwer Academic Publishers (2000) 37-59

6. Fukami,S., Mizumoto, M., Tanaka, T.: Some considerations on fuzzy conditional inference. Fuzzy Sets and Systems 4 (1981) 243-273

7. Klir, G.J, Yuan, B.: Fuzzy sets and fuzzy logic, theory and applications. Prentice Hall PTR (1995)

8. Sinha D., Dougherty, E.R.: Fuzzification of set inclusion: theory and applications. Fuzzy Sets and Systems 55 (1993) 15-42

9. Türkşen, I.B., Zhong, Z.: An approximate analogical reasoning scheme based on similarity measures and interval-valued fuzzy sets. Fuzzy Sets and Systems 34 (1990) 323-346

10. Van Der Donck, C.: A study of various fuzzy inclusions. Masters' thesis (in Dutch). University of Ghent (1998)

11. Van Der Donck, C., Kerre, E.E.: Sinha-Dougherty approach to the fuzzification of set inclusion revised. Submitted to Fuzzy Sets and Systems (2000)

12. Willmott, R.: Mean measures of containment and equality between fuzzy sets. Proc. of the 11th Int. Symp. on Multiple-Valued Logic, Oklahoma City (1981) 183-190

13. Young, V.R.: Fuzzy subsethood. Fuzzy Sets and Systems 77 (1996) 371-384

14. Zadeh, L.A.: Similarity relations and fuzzy orderings. Information Sciences 3 (1971) $177-200$ 